\title{
LINEAR RATIONAL FINITE DIFFERENCES FROM DERIVATIVES OF BARYCENTRIC RATIONAL INTERPOLANTS*
}

\author{
GEORGES KLEIN $^{\dagger}$ AND JEAN-PAUL BERRUT ${ }^{\dagger}$
}

\begin{abstract}
Derivatives of polynomial interpolants lead in a natural way to approximations of derivatives of the interpolated function, e.g., through finite differences. We extend a study of the approximation of derivatives of linear barycentric rational interpolants and present improved finite difference formulas arising from these interpolants. The formulas contain the classical finite differences as a special case and are more stable for calculating one-sided derivatives as well as
\end{abstract} derivatives close to boundaries. ences

Key words. linear rational interpolation, barycentric form, high order derivatives, finite differ-

AMS subject classifications. 65D05, 65L12, 41A05, 41A20, 41A25

1. Introduction: Linear barycentric rational interpolation. Suppose we are given a complex-valued function $f$ defined on an interval $I=[a, b]$ of the real line and distinct real values (nodes) $a=x_{0}<x_{1}<\cdots<x_{n}=b$. Let $f_{i}:=f\left(x_{i}\right)$, $i=0, \ldots, n$, be the given data and let $P_{n}[f]$ be the unique polynomial of degree at most $n$ which interpolates $f$ between the nodes, i.e., with $P_{n}[f]\left(x_{i}\right)=f_{i}$ for $i=0, \ldots, n$. The steps from the Lagrangian representation to its barycentric form

$$
P_{n}[f](x)=\sum_{i=0}^{n} \frac{\beta_{i}}{x-x_{i}} f_{i} / \sum_{i=0}^{n} \frac{\beta_{i}}{x-x_{i}},
$$

where the so-called weights $\beta_{i}$ are defined by

$$
\beta_{i}=\left[\prod_{j \neq i}\left(x_{i}-x_{j}\right)\right]^{-1}, \quad i=0, \ldots, n,
$$

are explained in many articles, such as [4, 7]. For details on how to easily implement this interpolant and for explicit $O(n)$ formulas for particular point sets, see [7].

Formula (1.1) allows an easy transition from polynomial to barycentric rational interpolation. Looking the right way at that equation and following [4], we see that $P_{n}[f]$ interpolates the data $f_{0}, \ldots, f_{n}$ at the nodes, no matter what the weights are, as long as these are all nonzero. If we replace the $\beta_{i}$ by other nonzero weights $\mu_{i}$, (1.1) becomes the general linear barycentric rational interpolant

$$
r_{n}[f](x)=\sum_{i=0}^{n} \frac{\mu_{i}}{x-x_{i}} f_{i} / \sum_{i=0}^{n} \frac{\mu_{i}}{x-x_{i}},
$$

the numerator and denominator of which are now polynomials of degree at most $n$. Every set of $n+1$ nonzero weights thus defines such a linear rational interpolant. In

\footnotetext{
†Department of Mathematics, University of Fribourg, Pérolles, 1700 Fribourg, Switzerland (georges.klein@unifr.ch, jean-paul.berrut@unifr.ch).
} 
[4], the second author of the present paper studied the simple choice

$$
\mu_{i}=(-1)^{i} \delta_{i}, \quad \delta_{i}:= \begin{cases}1 / 2, & i=0 \text { or } i=n, \\ 1 & \text { otherwise }\end{cases}
$$

The corresponding interpolant has no real poles and numerical experiments revealed that the error decreases like $1 / n^{2}$ for large $n$; see $[3,4]$. This choice of weights has been extended by Floater and Hormann in [13]. For every fixed nonnegative integer $d \leq n$, these authors considered the set of polynomials $p_{i}(x), i=0, \ldots, n-d$, interpolating $f$ at $x_{i}, \ldots, x_{i+d}$ and constructed the rational interpolant

$$
r_{n}[f](x)=\frac{\sum_{i=0}^{n-d} \lambda_{i}(x) p_{i}(x)}{\sum_{i=0}^{n-d} \lambda_{i}(x)},
$$

where

$$
\lambda_{i}(x):=\frac{(-1)^{i}}{\left(x-x_{i}\right) \cdots\left(x-x_{i+d}\right)} .
$$

They also found explicit formulas for the interpolation weights $\mu_{i}$ of the barycentric representation of $r_{n}$. To specify the interpolant we are studying, we shall denote this particular choice of weights by $w_{i}$. The approximation rate for a function $f \in$ $C^{d+2}[a, b]$ is $O\left(h^{d+1}\right)$ as $h \rightarrow 0$, where

$$
h:=\max _{0 \leq i \leq n-1}\left(x_{i+1}-x_{i}\right) .
$$

One advantage of these interpolants is the fact that the interpolation error depends on the maximum norm of just the $(d+2)$ nd order derivative of $f$, as opposed to the dependence on the $(n+1)$ st derivative in the polynomial case. (The latter is the particular case $d=n$.)

In the recent paper [5], the convergence rates of derivatives of this family of barycentric rational interpolants were investigated. The authors showed that the $k$ th derivative of the interpolant, as a function of the mesh size $h$, converges at the rate $O\left(h^{d+1-k}\right)$ in the cases $k=1,2$. The same convergence order was conjectured for $k=$ $3, \ldots, d$, at least for equispaced nodes. In section 2 we show that this conjecture holds true for the error at the nodes if these are equispaced or quasi-equispaced. For the approximation of higher order derivatives $(k \geq 3)$ at intermediate points, we suggest an even cheaper alternative, which yields convergence rates close to $O\left(h^{d+1-k}\right)$. In section 3 we present an important application of the convergence results, namely the derivation of linear barycentric rational finite differences. We conclude with some numerical examples in section 4.

2. Convergence rates of higher order derivatives at equispaced and quasi-equispaced nodes. In this section, we investigate the convergence rates of the $k$ th derivative, $k=1, \ldots, d$, of $r_{n}[f]$ from (1.4) at equispaced and quasi-equispaced nodes. Moreover, we consider an alternative method for the approximation of high order derivatives at intermediate points. By quasi-equispaced nodes [11] we shall mean here points whose minimal spacing $h_{\min }$ satisfies

$$
h_{\min } \geq c h,
$$

where $c$ is some positive constant less than 1 . 
Let us begin by recalling some notations from $[13,5]$. The interpolation error for $f \in C^{d+2}[a, b]$ may be rewritten as

$$
e(x)=\frac{\sum_{i=0}^{n-d}(-1)^{i} f\left[x_{i}, \ldots, x_{i+d}, x\right]}{\sum_{i=0}^{n-d} \lambda_{i}(x)} .
$$

We shall denote the numerator of $e(x)$ in the above expression by $A(x)$. As we concentrate on the derivatives of the error at a node $x_{j}$, we look at

$$
q_{j}(x):=\frac{e(x)}{x-x_{j}},
$$

which, evaluated at $x=x_{j}$, equals $e^{\prime}\left(x_{j}\right)$ by the definition of the derivative and the fact that $e\left(x_{j}\right)=0$. The properties of $q_{j}\left(x_{j}\right)$ and $q_{j}^{\prime}\left(x_{j}\right)$ have already been studied in [5]. We also define the functions

$$
\begin{aligned}
B_{j}(x) & :=\sum_{i \in I_{j}}(-1)^{i} \prod_{k=i, k \neq j}^{i+d} \frac{1}{x-x_{k}}, \\
C_{j}(x) & :=\sum_{i \in I \backslash I_{j}}(-1)^{i} \prod_{k=i}^{i+d} \frac{1}{x-x_{k}}
\end{aligned}
$$

where $I=\{0,1, \ldots, n-d\}$ and $I_{j}=\{i \in I: j-d \leq i \leq j\}$. With these notations we may rewrite $q_{j}(x)$ as

$$
q_{j}(x)=\frac{A(x)}{g_{j}(x)}
$$

with

$$
g_{j}(x):=B_{j}(x)+\left(x-x_{j}\right) C_{j}(x) .
$$

Comparing coefficients in the Taylor expansions of $e(x)$ and $q_{j}(x)$ at $x=x_{j}$, namely

$$
\begin{aligned}
e(x) & =\left(x-x_{j}\right) e^{\prime}\left(x_{j}\right)+\frac{1}{2 !}\left(x-x_{j}\right)^{2} e^{\prime \prime}\left(x_{j}\right)+\frac{1}{3 !}\left(x-x_{j}\right)^{3} e^{\prime \prime \prime}\left(x_{j}\right)+\cdots, \\
q_{j}(x) & =q_{j}\left(x_{j}\right)+\left(x-x_{j}\right) q_{j}^{\prime}\left(x_{j}\right)+\frac{1}{2 !}\left(x-x_{j}\right)^{2} q_{j}^{\prime \prime}\left(x_{j}\right)+\cdots,
\end{aligned}
$$

yields with $(2.1)$

$$
e^{(k)}\left(x_{j}\right)=k q_{j}^{(k-1)}\left(x_{j}\right), \quad k=1, \ldots, d .
$$

We use the following short notation introduced in [5]:

$$
d_{i k}:=\left|x_{i}-x_{k}\right| .
$$

TheOREM 2.1. Suppose $n, d, d \leq n$, and $k, k \leq d$, are positive integers and $f \in C^{d+1+k}[a, b]$. If the nodes $x_{j}, j=0, \ldots, n$, are equispaced or quasi-equispaced, then

$$
\left|e^{(k)}\left(x_{j}\right)\right| \leq C h^{d+1-k}, \quad 0 \leq j \leq n,
$$

where $C$ depends only on $c, d, k$, and derivatives of $f$. 
Proof. Throughout the development, we denote by $C$ any constant factor independent of $n$. For $k=1$ and $k=2$, the statement is covered by the general case of differentiation at arbitrarily distributed nodes; see Theorems 1 and 2 in [5].

We expand the derivatives of $q_{j}$ in $(2.3)$ by applying the Leibniz rule to the right-hand side of (2.2):

$$
q_{j}^{(k-1)}(x)=\sum_{\ell=0}^{k-1}\left(\begin{array}{c}
k-1 \\
\ell
\end{array}\right) A^{(k-1-\ell)}(x)\left(g_{j}^{-1}(x)\right)^{(\ell)} .
$$

Lemma 2 in [5] guarantees that the absolute value of every factor $A^{(k-1-\ell)}(x)$ is bounded in $[a, b]$ by a constant $C$; we thus look only at the last factor of the terms in the sum. We shall bound the $\ell$ th derivative of the reciprocal of $g_{j}(x)$ for $\ell=$ $0,1, \ldots, k-1$ at $x=x_{j}$. To this aim, we apply the "set partition version" of the Faà di Bruno formula for higher order derivatives of composite functions as given in [19] (see also $[10,12]$ ),

$$
\left(g_{j}^{-1}(x)\right)^{(\ell)}=\sum(-1)^{p} \frac{p !}{g_{j}^{p+1}(x)} \prod_{i=1}^{\ell}\left(g_{j}^{(i)}(x)\right)^{b_{i}},
$$

where the sum runs over all partitions of the set $\{1,2, \ldots, \ell\}$ and, for each partition, $p$ is its number of blocks and $b_{i}$ is the number of these blocks with precisely $i$ elements.

We shall use (2.6) to show (2.4) by means of a lower bound on $g_{j}\left(x_{j}\right)$ and an upper bound on the $\ell$ th derivative of $g_{j}(x)$ at $x=x_{j}$ for $\ell=1, \ldots, k-1$. The former has been explicitly established in [5] and is valid for any distribution of the nodes:

$$
\left|g_{j}\left(x_{j}\right)\right|=\left|B_{j}\left(x_{j}\right)\right| \geq \prod_{k=i, k \neq j}^{i+d} d_{j k}^{-1} \geq C h^{-d} \quad \forall i \in I_{j} .
$$

For the latter we consider

$$
g_{j}^{(\ell)}\left(x_{j}\right)=B_{j}^{(\ell)}\left(x_{j}\right)+\ell C_{j}^{(\ell-1)}\left(x_{j}\right)
$$

and use the Leibniz rule to obtain

$$
B_{j}^{(\ell)}(x)=\sum_{i \in I_{j}}(-1)^{i+\ell} \ell ! \sum_{\left|\mathbf{L}_{i, j}\right|=\ell} \prod_{k=i, k \neq j}^{i+d} \frac{1}{\left(x-x_{k}\right)^{1+\ell_{k-i}}},
$$

where $\mathbf{L}_{i, j}:=\left(\ell_{0}, \ldots, \ell_{j-i-1}, \ell_{j-i+1}, \ldots, \ell_{d}\right), j=0, \ldots, n$ and $i \in I_{j}$, are vectors whose components are nonnegative integers which sum up to

$$
\left|\mathbf{L}_{i, j}\right|:=\sum_{k=0, k \neq j-i}^{d} \ell_{k}
$$

Taking the absolute value of $B_{j}^{(\ell)}$ at $x=x_{j}$ leads to

$$
\left|B_{j}^{(\ell)}\left(x_{j}\right)\right| \leq \sum_{i \in I_{j}} \ell ! \sum_{\left|\mathbf{L}_{i, j}\right|=\ell} \prod_{k=i, k \neq j}^{i+d} d_{j k}^{-\left(1+\ell_{k-i}\right)} \leq C h^{-(d+\ell)},
$$


since every product in the inner sum involves the reciprocal of $d+\ell$ factors

$$
d_{j k} \geq|j-k| h_{\min } \geq C h .
$$

Analogously for $C_{j}$, with $\mathbf{L}:=\left(\ell_{0}, \ldots, \ell_{d}\right)$, it follows that

$$
C_{j}^{(\ell-1)}(x)=(\ell-1) ! \sum_{|\mathbf{L}|=\ell-1} \sum_{i \in I \backslash I_{j}}(-1)^{i+l-1} \prod_{k=i}^{i+d} \frac{1}{\left(x-x_{k}\right)^{1+\ell_{k-i}}} .
$$

We split the inner sum into its two parts with consecutive indices

$$
\sum_{i=0}^{j-d-1}(-1)^{i+l-1} \prod_{k=i}^{i+d} \frac{1}{\left(x-x_{k}\right)^{1+\ell_{k-i}}}+\sum_{i=j+1}^{n-d}(-1)^{i+l-1} \prod_{k=i}^{i+d} \frac{1}{\left(x-x_{k}\right)^{1+\ell_{k-i}}},
$$

where empty sums are meant to equal 0 . The terms in the left and right sums alternate in sign and increase and decrease, respectively, in absolute value. Therefore we obtain

$$
\left|C_{j}^{(\ell-1)}\left(x_{j}\right)\right| \leq(\ell-1) ! \sum_{|\mathbf{L}|=\ell-1}\left(\prod_{k=j-d-1}^{j-1} d_{j k}^{-\left(1+\ell_{k-j+d+1}\right)}+\prod_{k=j+1}^{j+1+d} d_{j k}^{-\left(1+\ell_{k-j-1}\right)}\right)
$$

which is bounded by a constant times $h^{-d-\ell}$, in the same fashion as (2.9). This result and (2.9) again, inserted into (2.8), yield

$$
\left|g_{j}^{(\ell)}\left(x_{j}\right)\right| \leq C h^{-(d+\ell)}, \quad \ell=1, \ldots, k-1 .
$$

Finally we show by induction that for $\ell=1, \ldots, k-1$,

$$
\left|\left(g_{j}^{-1}\left(x_{j}\right)\right)^{(\ell)}\right| \leq C h^{d-\ell} .
$$

For $\ell=0$ and $\ell=1$, this has been established in Theorems 1 and 2 in [5]. Now suppose that (2.11) holds for a certain $\ell$. Following Johnson's proof of the set partition version of the Faà di Bruno formula in [19], we use the form (2.6) to facilitate the step from the $\ell$ th to the $(\ell+1)$ st derivative of the reciprocal of $g_{j}$. Differentiating $(2.6)$ adds terms to the sum which equal the former ones with one of the following two changes:

$$
\frac{1}{g_{j}^{p+1}(x)} \longrightarrow-(p+1) \frac{g_{j}^{\prime}(x)}{g_{j}^{p+2}(x)} \quad \text { or } \quad\left(g_{j}^{(i)}(x)\right)^{b_{i}} \longrightarrow b_{i}\left(g_{j}^{(i)}(x)\right)^{b_{i}-1} g_{j}^{(i+1)}(x) \text {. }
$$

Equations (2.7) and (2.10) show that the bound on the $(\ell+1)$ st derivative of the reciprocal of $g_{j}$ at $x=x_{j}$ includes an additional factor $1 / h$ as compared with the bound on the $\ell$ th derivative.

We now turn our attention to the intermediate points, i.e., the $x \in[a, b]$ that are not interpolation points. The $k$ th $(k \geq 3)$ order derivative of $f$ at such an $x$ may also be approximated by the $k$ th derivative of $r_{n}[f]$, evaluated at that point. However, if $x$ is not a node, the expressions given in [20] for $r_{n}^{(k)}[f](x)$ as barycentric rational interpolants of divided differences get more and more expensive to evaluate and the formulas for the corresponding error $e^{(k)}(x)$ become very intricate. For this reason, and inspired by the polynomial case, we suggest that higher order derivatives of a function $f$ be approximated at intermediate points by the rational interpolant $R_{n}^{(k)}[f]$ 
of the approximations $r_{n}^{(k)}[f]\left(x_{i}\right)=: f_{i}^{(k)}$ of corresponding higher order derivatives at the nodes:

$$
R_{n}^{(k)}[f](x):=\sum_{i=0}^{n} \frac{w_{i}}{x-x_{i}} f_{i}^{(k)} / \sum_{i=0}^{n} \frac{w_{i}}{x-x_{i}} .
$$

In section 3, we shall recall and use elegant formulas for $f_{i}^{(k)}$ involving differentiation matrices. The following proposition shows that the maximum norm of the error,

$$
\left\|E^{(k)}\right\|=\max _{x \in[a, b]}\left|E^{(k)}(x)\right|, \quad E^{(k)}(x):=R_{n}^{(k)}[f](x)-f^{(k)}(x),
$$

decreases almost as $O\left(h^{d+1-k}\right)$ with an increasing number of equispaced or quasiequispaced nodes.

Proposition 2.2. Suppose $n, d, d \leq n$, and $k, k \leq d$, are positive integers and $f \in C^{d+2+k}[a, b]$. If the nodes $x_{j}, j=0, \ldots, n$, are equispaced or quasi-equispaced, then

$$
\left\|E^{(k)}\right\| \leq C h^{d+1-k}(1+\ln (n)),
$$

where $C$ depends only on $c, d, k$, and derivatives of $f$.

Proof. We again denote by $C$ any constant factor independent of $n$. As the error $\left|E^{(k)}\right|$ equals $\left|e^{(k)}\right|$ at the nodes, we need only consider intermediate points $x \neq x_{j}$. First, we see that $f^{(k)}$ belongs to $C^{d+2}[a, b]$ and may thus be interpolated by the rational function $r_{n}\left[f^{(k)}\right]$ with parameter $d$ and approximation rate $O\left(h^{d+1}\right)$, leading to

$$
\left|E^{(k)}(x)\right| \leq\left|R_{n}^{(k)}[f](x)-r_{n}\left[f^{(k)}\right](x)\right|+\left|r_{n}\left[f^{(k)}\right](x)-f^{(k)}(x)\right| .
$$

The first term may be bounded by

$$
\frac{\sum_{i=0}^{n}\left|\frac{w_{i}}{x-x_{i}}\right|\left|f_{i}^{(k)}-f^{(k)}\left(x_{i}\right)\right|}{\left|\sum_{i=0}^{n} \frac{w_{i}}{x-x_{i}}\right|}
$$

and the second by $C h^{d+1-k}$, using $h \leq(b-a)$. We have shown in Theorem 2.1 that

$$
\left|f_{i}^{(k)}-f^{(k)}\left(x_{i}\right)\right|=\left|e^{(k)}\left(x_{i}\right)\right| \leq C h^{d+1-k},
$$

so that (2.14) may be further bounded by

$$
C h^{d+1-k} \Lambda_{n}(x), \quad \text { where } \quad \Lambda_{n}(x)=\frac{\sum_{i=0}^{n}\left|\frac{w_{i}}{x-x_{i}}\right|}{\left|\sum_{i=0}^{n} \frac{w_{i}}{x-x_{i}}\right|}
$$

by definition, $\Lambda_{n}(x)$ is the Lebesgue function $[6,8,9]$ associated with the rational interpolant $r_{n}$. It is shown in [9] that the maximum $\Lambda_{n}$ of $\Lambda_{n}(x)$, i.e., the Lebesgue constant, is bounded by $2^{d-1}(2+\ln (n))$, independently of the interval length if the nodes are equispaced. And for quasi-equispaced nodes, in view of $c<1, \Lambda_{n}$ is bounded by $2^{d-1} / c^{d+1}(2 c+\ln (n))$ for $d \geq 1$ and by $3 /\left(4 c^{2}\right)(2 c+\ln (n))$ for $d=0$, as shown in [18]. The sum of the bounds on the first and second terms in (2.13) yields the claimed result.

Observe that the Lebesgue constant shows up in this proof. It coincides with the condition number of the interpolation process; see, e.g., [9]. This measure very naturally comes into play since $R_{n}^{(k)}[f]$ can be interpreted as the rational interpolant to the perturbed values $f_{i}^{(k)}$ of the derivatives $f^{(k)}\left(x_{i}\right)$ at the nodes.

In the next section we apply the results of Theorem 2.1 for constructing rational finite difference formulas. 
3. Linear barycentric rational finite difference (RFD) formulas. Finite difference (FD) methods based on polynomial interpolants have a long tradition and are still the subject of much investigation; see, for instance, $[14,15,16,17]$. The idea behind the corresponding formulas is the approximation of the $k$ th order derivative of $f$ at some point $\xi \in[a, b]$ by the $k$ th derivative of the polynomial interpolant $P_{n}[f]$ of $f$ at $\xi$. In view of the linearity of $P_{n}[f]$ in the data $f_{0}, \ldots, f_{n}$, an FD formula is obtained as follows:

$$
f^{(k)}(\xi) \approx P_{n}^{(k)}[f](\xi)=\sum_{j=0}^{n} c_{j} f_{j}
$$

For the determination of the weights $c_{j}$, Fornberg has presented a very efficient algorithm, which requires an average of four operations per weight. (Weights of finite differences must be distinguished from the weights of the barycentric form of rational interpolants.)

As an application of Theorem 2.1 and Proposition 2.2, we now introduce RFD formulas for the approximation of the $k$ th derivative of a sufficiently smooth function. For the approximation at a node $x_{i}$, we compute

$$
f^{(k)}\left(x_{i}\right) \approx r_{n}^{(k)}[f]\left(x_{i}\right)=\sum_{j=0}^{n} D_{i j}^{(k)} f_{j},
$$

where $D_{i j}^{(k)}$ is the $k$ th derivative at the node $x_{i}$ of the $j$ th Lagrange fundamental rational function. At an intermediate point $\xi \in[a, b]$, we consider

$$
f^{(k)}(\xi) \approx R_{n}^{(k)}[f](\xi)=\frac{\sum_{i=0}^{n} \frac{w_{i}}{\xi-x_{i}} f_{i}^{(k)}}{\sum_{i=0}^{n} \frac{w_{i}}{\xi-x_{i}}}=\sum_{j=0}^{n} \frac{\sum_{i=0}^{n} \frac{w_{i}}{\xi-x_{i}} D_{i j}^{(k)}}{\sum_{i=0}^{n} \frac{w_{i}}{\xi-x_{i}}} f_{j},
$$

which is also an FD formula, since the coefficients in the linear combination of the $f_{j}$ are constant for fixed $\xi$.

The methods presented here may be based on any linear barycentric rational interpolant. We nevertheless focus on the family of barycentric rational interpolants introduced recently in [13] and studied in section 2 .

In order to establish formulas for the weights $D_{i j}^{(k)}$ in $(3.1)$, we use the differentiation matrix $D^{(1)}$ with entries

$$
D_{i j}^{(1)}:= \begin{cases}\frac{w_{j}}{w_{i}} \frac{1}{x_{i}-x_{j}}, & i \neq j, \\ -\sum_{\ell=0, \ell \neq i}^{n} D_{i \ell}^{(1)}, & i=j\end{cases}
$$

defined in [3] for the first order derivative and similarly [21],

$$
D_{i j}^{(k)}:=\left\{\begin{array}{cc}
\frac{k}{x_{i}-x_{j}}\left(\frac{w_{j}}{w_{i}} D_{i i}^{(k-1)}-D_{i j}^{(k-1)}\right) & \\
=k\left(D_{i j}^{(1)} D_{i i}^{(k-1)}-\frac{D_{i j}^{(k-1)}}{x_{i}-x_{j}}\right), & i \neq j, \\
-\sum_{\ell=0, \ell \neq i}^{n} D_{i \ell}^{(k)}, & i=j,
\end{array}\right.
$$

for higher order derivatives. The formulas for these differentiation matrices were originally derived in $[1,3]$ from a result given by Schneider and Werner in [20]. The values on the diagonal, which guarantee exact derivatives of constants, are crucial for better accuracy [1]. 
TABLE 3.1

Weights for one-sided RFD formulas with $d=4$ and $n=4,5,6,7$ for the approximation of the first four derivatives at $x=0$ on an integer grid, $x_{0}=0, \ldots, x_{n}=n$.

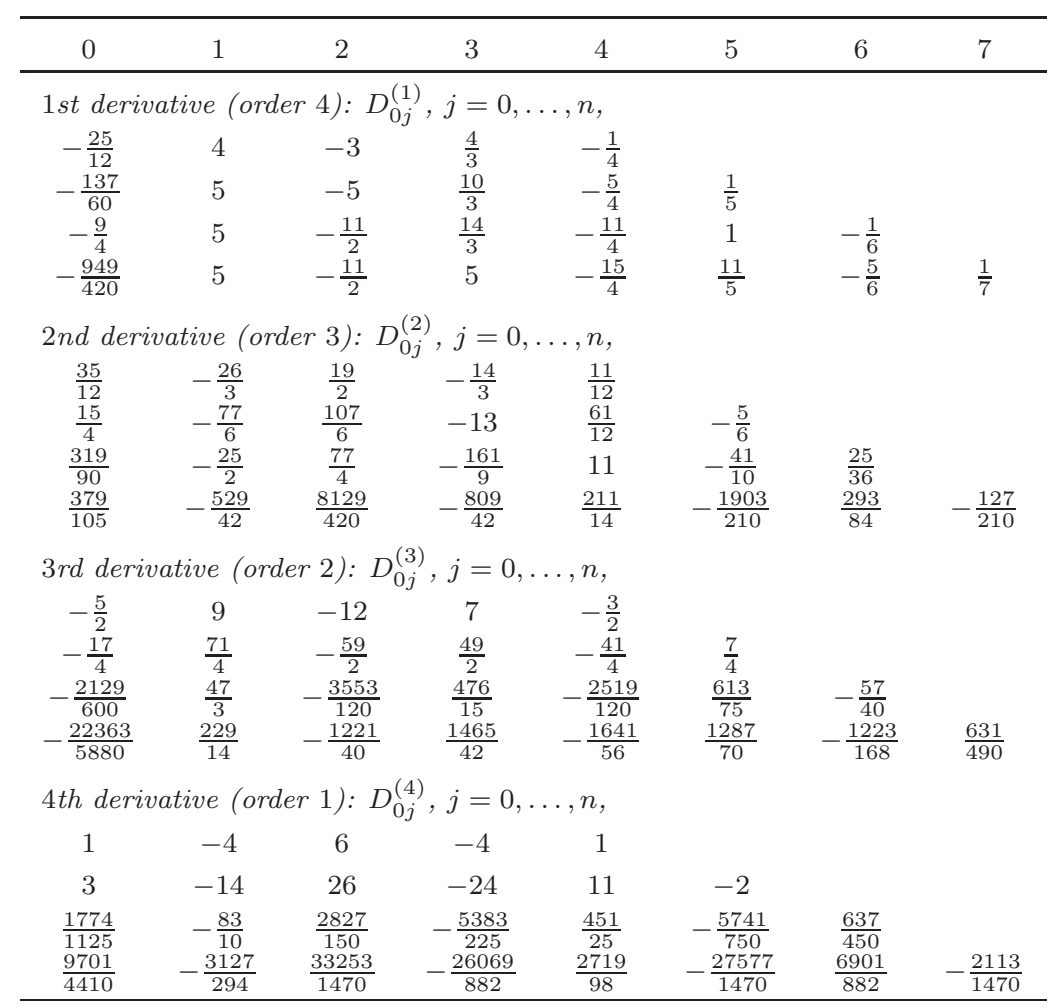

The weights for the approximation of the $k$ th derivative at the node $x_{i}$ are given by the entries of the $(i+1)$ st row of $D^{(k)}$. As each entry of the $i$ th row of $D^{(k)}$ depends only on three entries of the $i$ th row in $D^{(1)}$ and $D^{(k-1)}$, the computation of every additional RFD weight will require an average of four operations, as in the polynomial case.

In Tables 3.1 and 3.2, we list the weights for the first left one-sided $(i=0)$ and centered $(i=n / 2)$ RFD formulas on an equispaced grid for $d=4$ in (1.4), with a mesh size $h=1$. With another spacing, the weights must be divided by $h^{k}$. Since the differentiation matrices are centro-skew-symmetric for odd $k$ and centro-symmetric for even $k$, the weights of the right one-sided RFD formulas are the same as those of the left one-sided RFD $(i=n)$, though taken from right to left and multiplied by $(-1)^{k}$. Observe that for $n=d$ the RFD weights are the same as the FD weights. This is due to the fact that, in this special case, the rational interpolant $r_{n}[f]$ from (1.4) is the polynomial interpolant. Polynomial FD formulas indeed are a special case of their rational analogues.

Figures 3.1 and 3.2 show the absolute values of the weights of the first one-sided and centered RFD formulas with $d=3$ for the approximation of the first derivative at $x=0$ on an equispaced grid. Let us discuss these values and compare them with those obtained from polynomials in [15]; see Figures 3.2-1 and 3.2-2 in that reference.

The difference between polynomial FD and RFD is most striking with one-sided FD weights. In the polynomial case, the weights grow exponentially at the center of 
TABLE 3.2

Weights for centered RFD formulas with $d=4$ and $n=4,6,8$ for the approximation of the first four derivatives at $x=0$ on an integer grid, $x_{0}=-n / 2, \ldots, x_{n}=n / 2$.

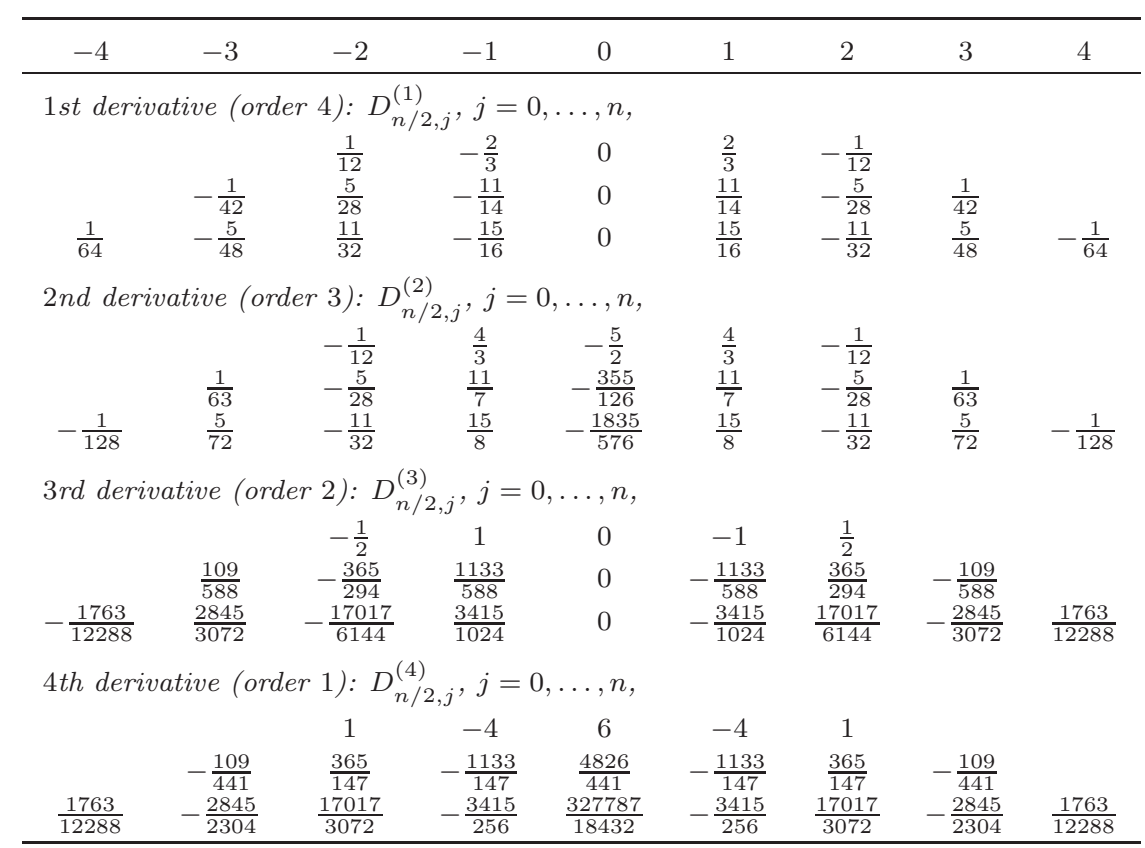

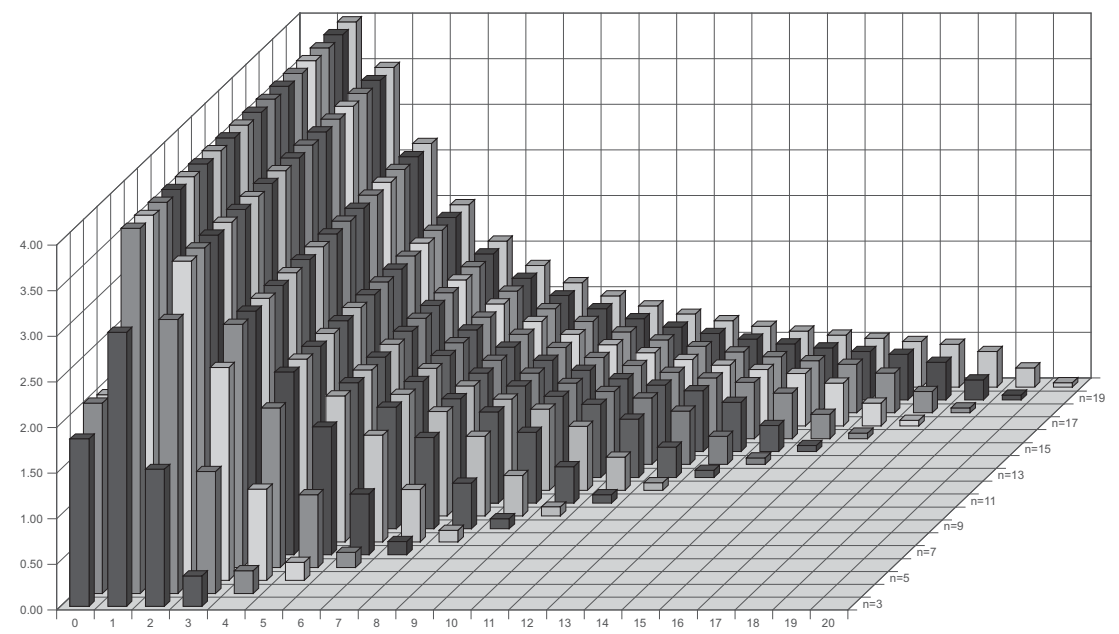

FIG. 3.1. Absolute values of the weights for one-sided RFD formulas with $d=3$ and $n=$ $3, \ldots, 20$ for the approximation of the first derivative at $x=0$ on an integer grid, $x_{0}=0, \ldots, x_{n}=n$.

the interval with increasing order or number of points [15]. The weights presented here behave much more favorably. To see this, we recall that the quotient of the largest to the smallest Floater-Hormann interpolation weight [13] is less than or equal to $2^{d}$ if the nodes are equispaced. Equation (3.2) shows that the one-sided RFD weights for the approximation of the first derivative oscillate in sign and decrease in absolute 


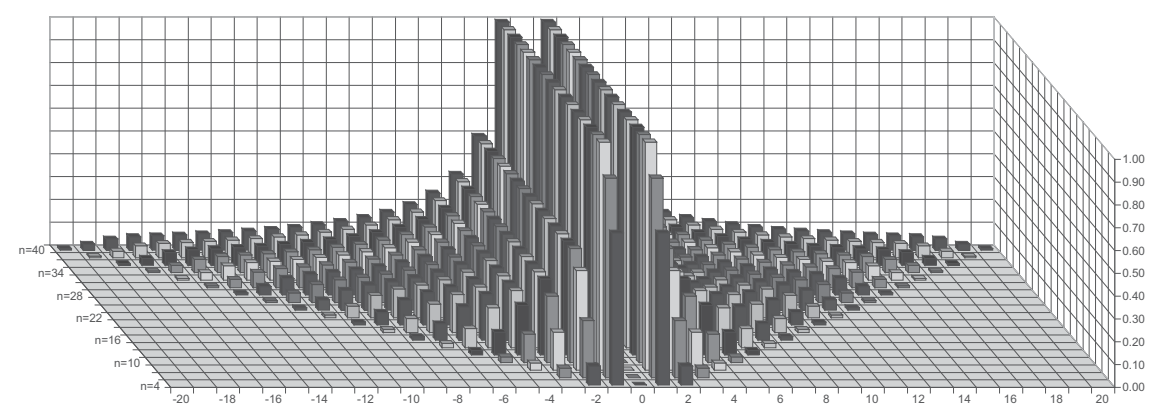

FIG. 3.2. Absolute values of the weights for centered RFD formulas with $d=3$ and $n=4,6, \ldots, 40$ for the approximation of the first derivative at $x=0$ on an integer grid, $x_{0}=-n / 2, \ldots, x_{n}=n / 2$.

value (relatively) at least as $1 /(i-j)$, where $i$ is the index of the maximal weight. Thus, the weight $D_{00}^{(1)}$ is neither the largest nor the smallest. This yields the following property:

$$
\frac{1}{b-a}<\left|D_{0 j}^{(1)}\right|<2^{d}, \quad j=0, \ldots, n,
$$

for every $n$ and $d, d \leq n$. For the approximation of higher order derivatives, (3.3) shows that the maximal weight is roughly bounded by $O\left(k ! 2^{k d}\right)$ for every $n$ and the neighboring weights decrease again as $1 /(i-j)$ in absolute value. The rather small values of the weights influence positively the numerical stability of the computation of one-sided RFD approximations as compared to their polynomial analogues.

An additional advantage of one-sided RFD is the fact that the maximal weight has index less than $n / 2$. This is very favorable since, as the derivative of a function is a local property, it is not natural to give great importance to function values located too far away from the point of interest.

In the centered case, the RFD weights behave similarly to the polynomial ones. For the approximation of the first derivative, they are bounded by 1 and decrease in absolute value as $1 /(i-j)$ from the maximal weight with index $i$. For higher order derivatives, the RFD weights also decrease in absolute value, like $1 /(i-j)$ for odd and $1 /(i-j)^{2}$ for even order derivatives; see the first formula in (3.3) and observe that $D_{\frac{n}{2} \frac{n}{2}}^{(k)}=0$ when both $k$ and $n$ are even.

4. Numerical results. We illustrate Theorem 2.1 and the above observations concerning the weights involved in polynomial and rational FD approximation. To this end, we have investigated the approximation of the second and fourth order derivatives of Runge's original example $f_{1}(x):=1 /\left(1+x^{2}\right)$ at the nodes $x=-5$ and $x=0$, and the modified example $f_{2}(x):=1 /\left(1+25 x^{2}\right)$ at $x=0$. We sampled them both at odd numbers of equispaced points, $f_{1}$ in the interval $[-5,5]$, respectively $[0,5]$, for one-sided FD approximation, and $f_{2}$ in $[-5,5]$ for centered FD approximation. In the rational interpolant (1.4), we chose $d=4$, the minimal value according to Theorem 2.1, to guarantee decreasing errors in the RFD approximation of the fourth order derivative.

Our aim was to observe estimated approximation orders of RFD approximations and to compare the error behaviors of polynomial and rational FD. For every example 


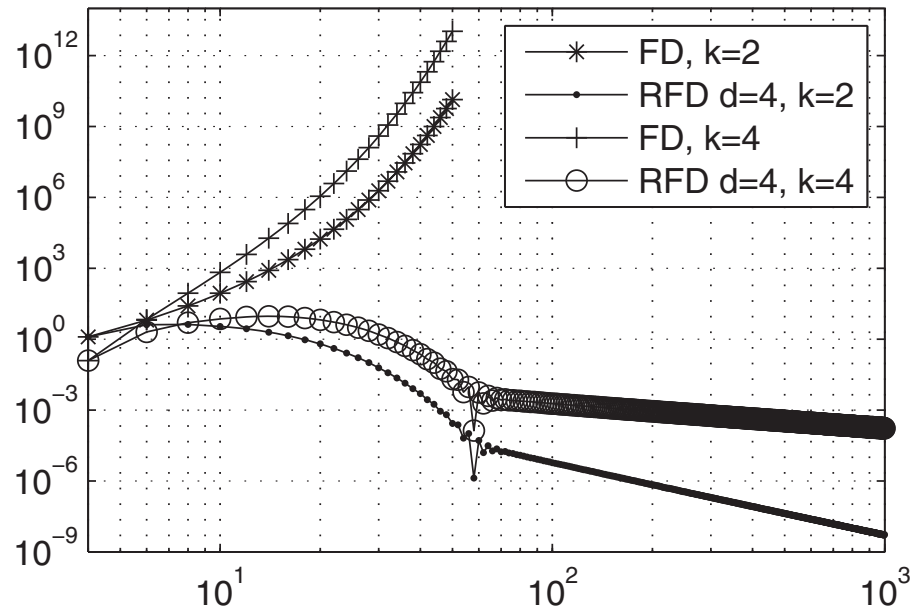

FIG. 4.1. Errors in the one-sided FD, respectively RFD, approximations at $x=-5$ (with $d=4$ and $n=4, \ldots, 1000)$ of the second and fourth order derivatives of $f_{1}$ sampled in $[-5,5]$.

we computed the absolute error in the various approximations. We performed this survey graphically on a log-log scale to avoid less informative tables and to display more details and data.

Figure 4.1 displays the absolute errors in one-sided polynomial FD and RFD approximations at $x=-5$ of the second $(k=2)$ and fourth $(k=4)$ order derivatives of $f_{1}$. The error in polynomial FD grows very rapidly with increasing values of $n$. This failure arises from Runge's phenomenon and the large growth rates of the polynomial FD weights [15]; for $n=140$, the largest absolute value of these weights for the approximation of the fourth order derivative is $7.6 \mathrm{e}+41$. In RFD with $d=4$, in contrast, the largest value is only 28.6. From the slopes of the curves, we see that the errors in RFD approximation of the second and fourth order derivatives of $f_{1}$ decrease at an experimental rate of 3 , respectively 1 , which is the lower bound $d+1-k$ predicted in Theorem 2.1. The good quality of approximation in the present example is also to be expected in RFD approximation at nodes near the ends of the interval.

In the next example, namely one-sided FD and RFD approximations at $x=0$ of the same derivatives of $f_{1}$ as above, Runge's phenomenon does not appear, only the bad conditioning of one-sided polynomial FD makes the error grow as $n$ becomes larger than 45; see Figure 4.2. The errors in RFD approximation are larger until this value of $n$, but, as $n$ increases, they keep decreasing at experimental rates of 4 , respectively 2 , which is one more unit than predicted by the upper bound (2.4) on the error.

We did a similar study with the analytic function $f(x)=\exp \left(-50(x-0.5)^{2}\right)$; namely we sampled it in $[0,1]$ and compared one-sided FD and RFD approximations at $x=0$ of the first and third derivatives for various values $\leq 6$ of $d$ and for $n$ between $d$ and 1000. The convergence rates of the RFD approximation from Theorem 2.1 are observable as soon as $n \geq 15$, whereas the relative error in polynomial FD is larger than one with small values of $n$ and starts diverging when $n$ exceeds 50 .

The absolute errors in centered polynomial FD and RFD approximations of the 


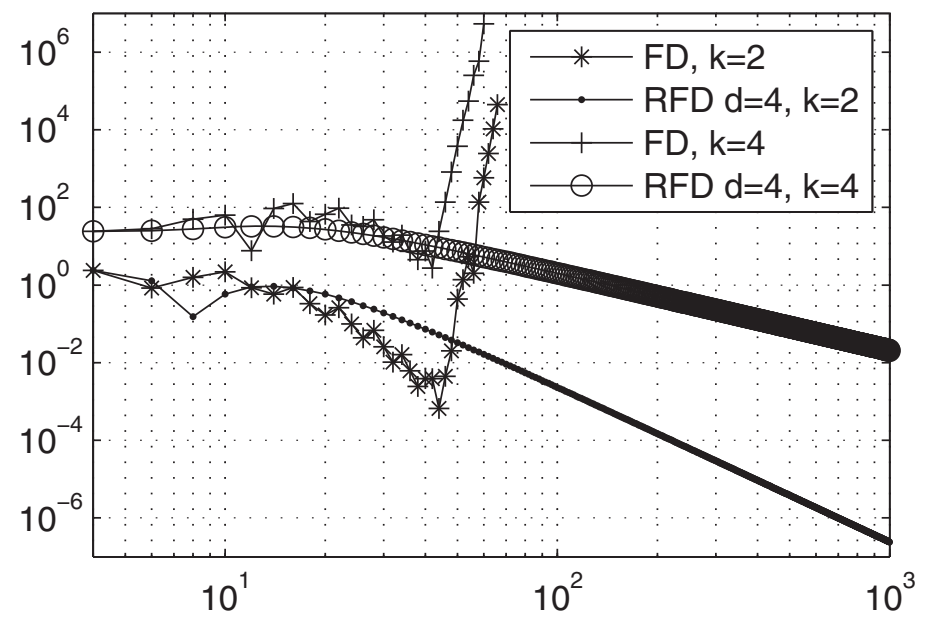

FIG. 4.2. Errors in the one-sided FD, respectively RFD, approximations at $x=0$ (with $d=4$ and $n=4, \ldots, 1000$ ) of the second and fourth order derivatives of $f_{1}$ sampled in $[0,5]$.

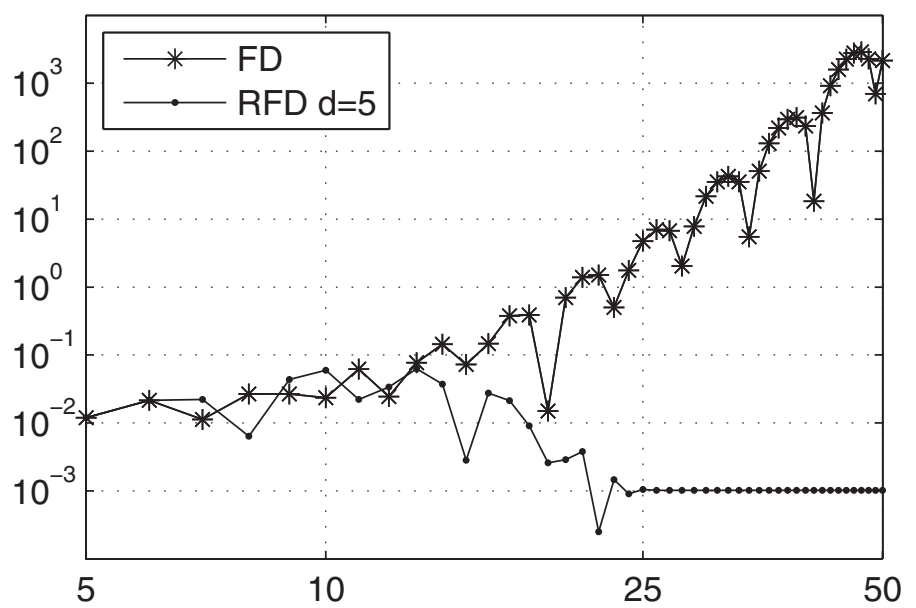

FIG. 4.3. Errors in the one-sided FD, respectively RFD, approximations at $x=0$ (with $d=5$ and $n=5, \ldots, 50)$ of the first order derivative of $\exp \left(-50(x-0.4)^{2}\right)+\sinh (x)$ sampled in $[0,0.04 n]$.

second and fourth order derivatives of $f_{2}$ at $x=0$ nearly coincide. We thus omit plotting them. While the error in polynomial FD decays exponentially, as expected since there is no Runge phenomenon in the middle of the interval, the error in the RFD approximation decreases much faster than predicted in this particular example for $n$ between 50 and 450. For larger values of $n$, the absolute errors start oscillating. Other examples with centered RFD yield the expected algebraic decay of the error as the number of nodes increases, while the errors in polynomial centered FD always decayed exponentially for smooth functions.

Another approach to FD approximation is to keep $n$ constant and small, and 
decrease $h$ as much as needed to reach the desired precision. In this setting, the classical FD methods are best since their weights have roughly the same magnitude, the Runge phenomenon disappears due to the shrinkage of the interval - the ellipses, where the function needs to be analytic for the polynomial interpolant to converge, shrink - and every reasonable continuous function can be represented by a polynomial of small degree in a short interval.

Finally we performed the converse experiment: we chose $h$ fixed and increased $n$, a situation which may arise when the function cannot be sampled with arbitrarily small resolution. The first derivative of $f(x)=\exp \left(-50(x-0.4)^{2}\right)+\sinh (x)$ was approximated at $x=0$ using $n+1$ function values in the interval $[0,0.04 n]$. Increasing the number of nodes and thus the information about the function does not help to gain any precision when using polynomial FD methods; see Figure 4.3. With RFD and the proper choice of $d$, it was possible to further decrease the error in the approximation of the derivative in this example. However, the determination of the optimal $d$ is often a nontrivial task.

5. Conclusion. We have established bounds on the error of the approximation of higher order derivatives at equispaced and quasi-equispaced nodes using a family of linear barycentric rational interpolants. As an application, we have presented rational finite difference (RFD) formulas, an extension of classical finite difference (FD) methods, which are based on polynomial interpolation. The magnitudes of the weights in the RFD formulas reveal that the latter are more stable, especially in onesided approximations and near the ends of the interval considered. The numerical examples confirm this fact, which could be of considerable importance when using FD approximations of (partial) derivatives in the vicinity of domain boundaries.

\section{REFERENCES}

[1] R. Baltensperger And J.-P. Berrut, The errors in calculating the pseudospectral differentiation matrices for Čebyšev-Gauss-Lobatto points, Comput. Math. Appl., 37 (1999), pp. $41-48$.

[2] R. BAltensperger And J.-P. Berrut, Errata to: "The errors in calculating the pseudospectral differentiation matrices for Čebyšev-Gauss-Lobatto points", Comput. Math. Appl., 38 (1999), p. 119.

[3] R. Baltensperger, J.-P. Berrut, And B. Nö̈L, Exponential convergence of a linear rational interpolant between transformed Chebyshev points, Math. Comp., 68 (1999), pp. 1109-1120.

[4] J.-P. BERRUT, Rational functions for guaranteed and experimentally well-conditioned global interpolation, Comput. Math. Appl., 15 (1988), pp. 1-16.

[5] J.-P. Berrut, M. S. Floater, and G. Klein, Convergence rates of derivatives of a family of barycentric rational interpolants, Appl. Numer. Math., 61 (2011), pp. 989-1000.

[6] J.-P. BerRut And H. D. MitTelmann, Lebesgue constant minimizing linear rational interpolation of continuous functions over the interval, Comput. Math. Appl., 33 (1997), pp. 77-86.

[7] J.-P. Berrut And L. N. Trefethen, Barycentric Lagrange interpolation, SIAM Rev., 46 (2004), pp. 501-517.

[8] L. Bos, S. De Marchi, And K. Hormann, On the Lebesgue constant of Berrut's rational interpolant at equidistant nodes, J. Comput. Appl. Math., 236 (2011), pp. 504-510.

[9] L. Bos, S. De Marchi, K. Hormann, and G. Klein, On the Lebesgue constant of barycentric rational interpolation at equidistant nodes, Numer. Math., to appear.

[10] L. Comtet, Advanced Combinatorics, D. Reidel, Dordrecht, The Netherlands, 1974.

[11] V. Elling, A Lax-Wendroff type theorem for unstructured quasi-uniform grids, Math. Comp., 76 (2007), pp. 251-272.

[12] H. Flanders, From Ford to Fà̀, Amer. Math. Monthly, 108 (2001), pp. 559-561.

[13] M. S. Floater And K. Hormann, Barycentric rational interpolation with no poles and high rates of approximation, Numer. Math., 107 (2007), pp. 315-331.

[14] B. FornberG, Generation of finite difference formulas on arbitrary spaced grids, Math. Comp., 51 (1988), pp. 699-706. 
[15] B. Fornberg, A Practical Guide to Pseudospectral Methods, Cambridge University Press, Cambridge, UK, 1996.

[16] B. Fornberg, Calculation of weights in finite difference formulas, SIAM Rev., 40 (1998), pp. $685-691$.

[17] B. Fornberg And D. M. Sloan, A review of pseudospectral methods for solving partial differential equations, in Acta Numer., Cambridge University Press, Cambridge, UK, 1994, pp. 203-267.

[18] K. Hormann, G. Klein, and S. De Marchi, Barycentric rational interpolation at quasiequidistant nodes, Dolomites Res. Notes Approx., 5 (2012), pp. 1-6.

[19] W. P. Johnson, The curious history of Faà di Bruno's formula, Amer. Math. Monthly, 109 (2002), pp. 217-234.

[20] C. Schneider and W. Werner, Some new aspects of rational interpolation, Math. Comp., 47 (1986), pp. 285-299.

[21] W. TeE, An Adaptive Rational Spectral Method for Differential Equations with Rapidly Varying Solutions, Ph.D. thesis, University of Oxford, Oxford, UK, 2006. 